\title{
Comparing the interobserver reproducibility of different regions of interest on multi-parametric renal magnetic resonance imaging in healthy volunteers, patients with heart failure and renal transplant recipients
}

\author{
Alastair J. Rankin ${ }^{1}$ (D) Sarah Allwood-Spiers ${ }^{2} \cdot$ Matthew M. Y. Lee $^{1} \cdot$ Luke Zhu $^{1} \cdot$ Rosemary Woodward $^{3}$. \\ Bernd Kuehn ${ }^{4} \cdot$ Aleksandra Radjenovic $^{1} \cdot$ Naveed Sattar $^{1} \cdot$ Giles Roditi $^{5} \cdot$ Patrick B. Mark ${ }^{1} \cdot$ Keith A. Gillis $^{1}$
}

Received: 12 July 2019 / Revised: 18 November 2019 / Accepted: 22 November 2019 / Published online: 10 December 2019

(C) The Author(s) 2019

\begin{abstract}
Objective To assess interobserver reproducibility of different regions of interest (ROIs) on multi-parametric renal MRI using commercially available software.

Materials and methods Healthy volunteers (HV), patients with heart failure (HF) and renal transplant recipients (Tx) were recruited. Localiser scans, T1 mapping and pseudo-continuous arterial spin labelling (pCASL) were performed. HV and Tx also underwent diffusion-weighted imaging to allow calculation of apparent diffusion coefficient (ADC). For T1, pCASL and ADC, ROIs were drawn for whole kidney (WK), cortex $(\mathrm{Cx})$, user-defined representative cortex (rep-Cx) and medulla. Intraclass correlation coefficient (ICC) and coefficient of variation $(\mathrm{CoV})$ were assessed.

Results Forty participants were included (10 HV, $10 \mathrm{HF}$ and $20 \mathrm{Tx}$ ). The ICC for renal volume was 0.97 and CoV 6.5\%. For T1 and ADC, WK, Cx, and rep-Cx were highly reproducible with ICC $\geq 0.76$ and $\mathrm{CoV}<5 \%$. However, cortical pCASL results were more variable (ICC $>0.86$, but $\mathrm{CoV}$ up to $14.2 \%$ ). While reproducible, WK values were derived from a wide spread of data (ROI standard deviation $17 \%$ to $55 \%$ of the mean value for ADC and pCASL, respectively). Renal volume differed between groups $(p<0.001)$, while mean cortical T1 values were greater in Tx compared to HV $(p=0.009)$ and HF $(p=0.02)$. Medullary T1 values were also higher in Tx than HV $(p=0.03)$, while medullary pCASL values were significantly lower in Tx compared to HV and HF ( $p=0.03$ for both).

Discussion Kidney volume calculated by manually contouring a localiser scan was highly reproducible between observers and detected significant differences across patient groups. For T1, pCASL and ADC, Cx and rep-Cx ROIs are generally reproducible with advantages over WK values.
\end{abstract}

Keywords Renal MRI $\cdot$ Reproducibility $\cdot$ Chronic kidney disease $\cdot$ Renal transplantation $\cdot$ Heart failure

\section{Introduction}

Electronic supplementary material The online version of this article (https://doi.org/10.1007/s10334-019-00809-4) contains supplementary material, which is available to authorized users.

Alastair J. Rankin

alastair.rankin@nhs.net

1 Room 311, Institute of Cardiovascular and Medical Sciences, BHF Glasgow Cardiovascular Research Centre, University of Glasgow, 126 University Place, Glasgow G12 8TA, UK

2 Department of Clinical Physics and Bioengineering, NHS Greater Glasgow and Clyde, Glasgow, UK
Functional renal imaging is a burgeoning field of research that has the potential to translate into meaningful clinical applications for patients with kidney disease [1].

Clinical Research Imaging, NHS Greater Glasgow and Clyde, Glasgow, UK

4 Siemens Healthcare GmbH, Erlangen, Germany

5 Department of Radiology, NHS Greater Glasgow and Clyde, Glasgow, UK 
Multi-parametric magnetic resonance imaging (MRI) allows acquisition of multiple sequences with potential to inform regarding structure, tissue composition, perfusion, and physiology of renal function in a single scan [2]. However, the clinical utility of each sequence, and indeed the potential additive benefit of their use together, are yet to be proven. The immediate research priority in renal MRI is focusing on the standardisation and harmonisation of image acquisition across research sites and MRI vendors. This 'ground-up' approach is driven by international, independently funded working groups, including PARENCHIMA [2], a subsidiary of the European Cooperation in Science and Technology (COST) Action group and the UK Renal Imaging Network (UKRIN), amongst others. As image acquisition is standardised, scientific scrutiny must also be applied to the methods of analysis. Many of the MRI sequences employed produce quantitative results from modelling dependent on measurements using other sequences [3], and for which the resultant values will vary depending on whether whole kidney, renal cortex or renal medulla is selected [4]. Numerous analytic approaches have been reported to date, and the optimal technique in terms of time and clinical relevance, are not yet known. In addition, the absence of commercially available analysis software that is specifically designed for unique interests of renal MRI leads to use of in-house bespoke software, which renders external validation of results challenging.

Our centre has an active renal MRI research group, with current projects exploring the clinical implications of multi-parametric renal MRI across healthy volunteers [5] as well as patients with heart failure, chronic kidney disease (CKD) [6] and renal transplants. We aim to compare different regions of interest (ROIs) and their interobserver reproducibility using commercially available analysis software in healthy and patient populations, including native and transplant kidneys, across selected MRI sequences.

\section{Methods}

\section{Study population and clinical parameters}

Patients were recruited from nephrology and cardiology clinics, and from general advertisement, for the renal transplant (Tx), heart failure (HF) and healthy volunteer (HV) cohorts, respectively. For Tx and HF patients, the scans were acquired as baseline imaging for two separate ongoing clinical studies (ClinicalTrials.gov: NCT03705091 and NCT03485092). Basic biometric parameters were measured and serum creatinine was measured in accredited clinical biochemical laboratories. Estimated glomerular filtration rate (eGFR) was derived using the Chronic Kidney Disease Epidemiology Collaboration (CKD-EPI) equation [7]. All participants gave written informed consent and regional ethics committee approval was granted; the study was conducted in agreement with the Declaration of Helsinki.

\section{MRI acquisition}

MRI was performed on a Siemens MAGNETOM Prisma 3T scanner (Siemens Healthcare, Erlangen, Germany) using an 18-channel phased array coil anteriorly and a 32-channel spine coil posteriorly. Scans for renal volume, perfusion and T1 were acquired from all patients (Fig. 1), with the transplant kidney scanned for the Tx group. Diffusion-weighted imaging (DWI) was performed on the Tx and HV cohorts. Patients were imaged supine.

- Volume Coronal images were acquired during a breath hold at expiration using a steady-state free precession sequence [true fast imaging with steady-state precession (TrueFISP)]. The imaging parameters used are listed in Supplementary Material Table 1 (HV and Tx cohorts) and Supplementary Material Table 2 (HF cohort).

- T1 T1 maps were acquired for a single coronal oblique slice through the centre of the kidney using a modified look-locker inversion recovery (MOLLI) $[8,9]$ sequence with single shot TrueFISP readout [10]. For Tx and HV cohorts, images were acquired at 14 different inversion times (TI) [acquisition scheme 11(3)3] with an initial delay of $180 \mathrm{~ms}$ after the first inversion pulse and a delay of $260 \mathrm{~ms}$, after the second inversion pulse. The interval between subsequent measurements was $550 \mathrm{~ms}$, resulting in TIs of 180, 260, 730, 810, 1280, 1360, 1830, 2380, 2930, 3480, 4030, 4580, 5130, $5680 \mathrm{~ms}$ ) and echo spacing of $3.04 \mathrm{~ms}$. The acquisition time was $10 \mathrm{~s}$. Images were acquired during free breathing.

For the HF cohort, images were acquired at eight different inversion times [acquisition scheme 5(3)3] with a start TI of $100 \mathrm{~ms}$, a TI increment of $80 \mathrm{~ms}$ (inversion times dependent on captured cardiac cycle), a reported TR of $280-340 \mathrm{~ms}$, and echo spacing of $2.44 \mathrm{~ms}$. Images were acquired during a breath hold. Other imaging parameters are given in Supplementary Materials Tables 1, 2.

Motion correction and fitting of the T1 map was performed using a phase-sensitive inversion recovery reconstruction implemented in the vendor software (Siemens, VE11C, MyoMaps) [11].

- Arterial spin labelling A pseudo-continuous arterial spin labelling (pCASL) scan [12] with a 3D turbo gradient spin-echo (TGSE) readout which was acquired during free breathing [13]. The prototype sequence comprises a slice-selective presaturation pulse to suppress the sig- 


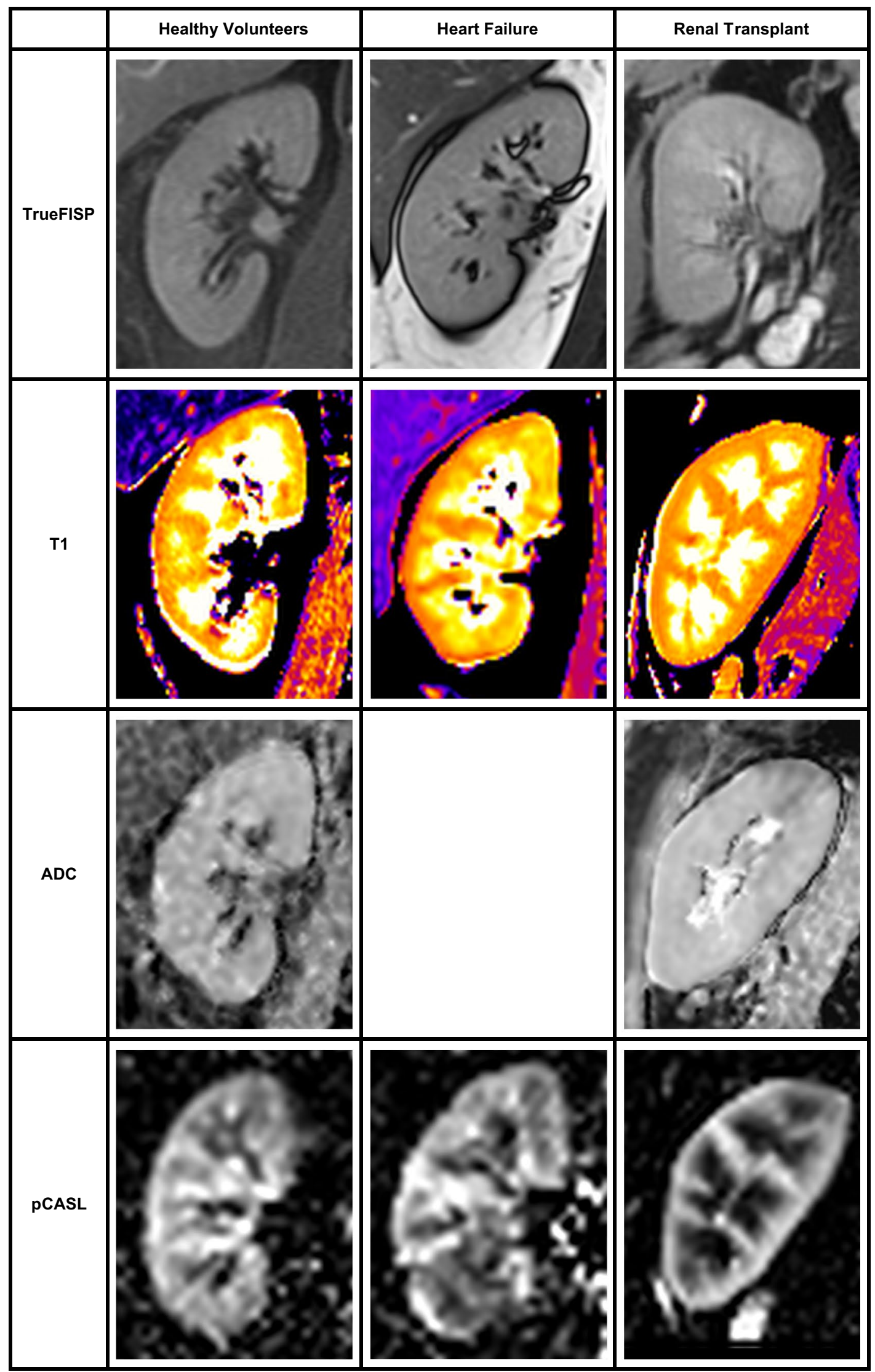

Fig. 1 Representative image of each MRI sequence for each participant group 
nal from preceding excitations and a frequency-offsetcorrected inversion (FOCI) pulse positioned over the imaging region. This is followed by the pCASL sliceselective labelling pulse. For background saturation, four non-selective hyperbolic secant pulses are applied, interspersed with three slice-selective saturation pulses, positioned superior to the labelling plane.

The pCASL labelling plane was positioned in a transverse oblique slice of thickness $10 \mathrm{~mm}$ perpendicular to the aorta and superior to the kidneys to label the blood in the descending aorta (Supplementary Material Fig. 1). The start time of the pCASL labelling was $3000 \mathrm{~ms}$ and the pCASL duration was $1500 \mathrm{~ms}$ with a flip angle of $28^{\circ}$. The presaturation pulses and FOCI pulse were positioned in a transverse slab covering the kidneys. The pulses to suppress inflowing arterial blood were applied in a slab superior to the labelling plane to suppress inflowing arterial blood. Images were obtained in a coronal oblique orientation covering the whole kidney volume. A low-resolution pCASL scan with one measurement was acquired to confirm that the positioning of the labelling plane was appropriate to produce signal in the perfusion-weighted image. This was followed by a higher resolution scan with parameters as given in Tables 1,2. The sequence acquires label and control images and a reference proton density-weighted (M0) image.

Perfusion maps were produced using inline software. In-plane 2D motion correction is applied, retrospectively, to proton density-weighted $\left(M_{0}\right)$ label and control images. Label and control images are subtracted to create perfusion-weighted images. Maps of perfusion rate $(f)$ are calculated pixel by pixel using the motion-corrected proton density-weighted $\left(M_{0}\right)$ and perfusion-weighted $(\Delta M)$ images according to:

$$
\begin{aligned}
\Delta M= & f \frac{2 M_{0}}{\lambda} T_{1^{\prime}} \alpha \exp \left(\frac{-\Delta t}{T_{1 \text { blood }}}\right) \\
& \exp \left(\frac{-(t-\tau-\Delta t)}{T_{1^{\prime}}}\right)\left(1-\exp \left(\frac{-\tau}{T_{1^{\prime}}}\right)\right),
\end{aligned}
$$

where $f$ is the perfusion rate in $\mathrm{ml} / 100 \mathrm{mg} / \mathrm{min}$; $t$ is the time between labelling and imaging $(3000 \mathrm{~ms}) ; \tau$ is the duration of labelling pulse ( $1500 \mathrm{~ms}) ; \Delta t$ is the arterial transit time, assumed to be $750 \mathrm{~ms}$; $\alpha$ is the labelling efficiency, assumed to be $0.98 ; \lambda$ is the blood-tissue water partition coefficient, assumed to be $0.9 \mathrm{ml} / 100 \mathrm{~g} ; T_{\text {blood }}$ is the longitudinal relaxation time of arterial blood; $T_{1^{\prime}}$ is the apparent longitudinal relaxation time of tissue. A fixed $T_{1 \text { blood }}=T_{1^{\prime}}=1250 \mathrm{~ms}$ was assumed in calculating the perfusion maps.

- DWI For the Tx and HV cohorts, DWI was performed using a single-shot spin-echo echo-planar imaging sequence with 17 slices positioned in a coronal oblique plane. Images were acquired at $10 b$ values $(0,50,100,150,200,250,300,500,750,1000 \mathrm{~s} /$ $\mathrm{mm}^{2}$ ) for four diffusion directions, averaged to give a 4-scan trace. Spectral attenuated inversion recovery (SPAIR) fat suppression was used and images were acquired during free breathing, with an acquisition time of 1 min $46 \mathrm{~s}$. Apparent diffusion coefficient (ADC) maps were created using the vendor software, performing a mono-exponential fit to the ten $b$-values [14].

\section{MRI analysis}

Interobserver variability was compared across different methods of image analysis. For kidney volume, the renal contours were drawn around the whole kidney (excluding the renal pelvis) on the first and last slices containing renal tissue. Contours were then added to every alternate slice in between. This initial total kidney volume (linear interpolation for non-contoured slices) was then recorded ('alternate slices') prior to drawing contours to the remaining slices and noting the resultant volume ('every slice'). For pCASL and DWI, a single slice was chosen for analysis. ROIs were drawn manually around the whole kidney (WK), cortex $(\mathrm{Cx})$, an area of user-defined representative cortex (rep-Cx), within the cortex at the superior and inferior poles (sup$\mathrm{Cx}$ and inf-Cx, respectively) and in a representative area of medulla (Med) (Fig. 2). Corticomedullary differentiation was assessed by ratio of $\mathrm{Cx}$ to Med. Each cohort was analysed by a pair of independent observers from a pool of four clinicians and one physicist, all with local training in renal MRI analysis (SAS and LZ analysed HV, SAS and MMYL analysed HF and KAG and AJR analysed Tx). Image analysis was performed using the commercially available software cvi42 version 5.9.4 (Circle Cardiovascular Imaging, Calgary, Canada).

\section{Statistical analysis}

Descriptive statistics are reported as mean and standard deviation or median and range/interquartile range (IQR) for normally distributed and skewed data, respectively. Paired t-tests were used to compare kidney volume techniques and results were displayed graphically using a Bland-Altman plot [15]. Pearson correlation coefficient was used to quantify linear relationships between continuous variables. A total of 12 participants are required to detect a correlation coefficient of 0.8 with $90 \%$ power and alpha 0.05 . Our decision to include 40 participants yields a power $>99.9 \%$ to detect a correlation coefficient of 0.8 at alpha 0.05 . Interobserver reproducibility was measured using coefficient of variation $(\mathrm{CoV})$ (calculated by the standard deviation divided by the mean) and intraclass correlation coefficient (ICC) 
(two-way random, average measures). One-way ANOVA was used to compare mean results across the three participant groups, with t-tests to interrogate pairs where groups differed. The mean value of the two observers is reported unless otherwise stated. All analyses were performed using SPSS Statistics Version 25.0 (Armonk, NY: IBM Corp.) and a conventional significance level of $<0.05$ was used. Figures were generated using SPSS Statistics Version 25.0 (Armonk, NY: IBM Corp.) and Microsoft PowerPoint ${ }^{\circledR} 2019$.

\section{Results}

\section{Participant demographics}

A total of 40 participants were included: ten healthy volunteers, ten patients with heart failure (with reduced ejection fraction of $\leq 40 \%$ ) and 20 renal transplant recipients. Clinical characteristics are shown in Table 1.

\section{Renal volume}

Calculation of renal volume was possible in 39 patients (98\%) (one patient did not have appropriate TrueFISP images). Mean difference in renal volume was $1.6 \mathrm{ml}$ lower when contours were drawn on alternate slices as opposed to every slice $(p<0.001)$ (Fig. 3$)$. There was no interobserver difference in renal volume with either approach ( $p=0.56$ for alternate slice, and $p=0.89$ for every slice). Tables 2, 3 show the results and interobserver reproducibility for renal volume, respectively.

\section{T1, pCASL, ADC: comparison of different ROIs}

T1, pCASL and ADC sequences were acquired in 39, 39 and 28 patients, respectively. Image quality was acceptable in all but two pCASL acquisitions in the Tx group who were excluded from further analysis. Table 2 shows the mean results for each sequence depending on whether ROIs were drawn for WK, Cx, rep-Cx, sup-Cx, inf-Cx and Med. The standard deviation in Table 2 represents the spread of mean values obtained. Table 3 shows the interobserver reproducibility for each ROI by sequence and participant group. For $\mathrm{T} 1$ and $\mathrm{ADC}, \mathrm{WK}, \mathrm{Cx}$ and rep-Cx were highly reproducible ( $\mathrm{ICC} \geq 0.76$; $\mathrm{CoV}<5 \%$ ). For pCASL, Cx and rep-Cx were less readily reproducible (ICC $>0.86$ but $\mathrm{CoV}$ up to $14.2 \%)$. The reproducibility of Med ROI was excellent for T1, but less good for pCASL and ADC (Table 3). Table 4 shows the spread of data within each ROI by reporting the mean ROI standard deviation as a proportion of the mean value. The spread of data from WK ROIs was higher than cortex-specific ROIs, even when the mean value for each was similar (Table 2).

\section{Correlation between different ROls}

For T1, the correlation coefficient for Cx compared to WK, rep-Cx, sup-Cx, inf-Cx and Med was 0.76, 0.93, 0.86, 0.85 and 0.62 , respectively. The corresponding values for pCASL were 0.92, 0.91, 0.84, 0.81 and 0.26; and for ADC 0.87, $0.79,0.75,0.85$ and $0.78[p<0.001$ for all, except pCASL Med which was not significant $(p=0.13)]$.

\section{Comparison between participant groups}

There was a significant difference in kidney volume between groups $(F=13.2, p<0.001)$ with the greatest renal volume in Tx, then HF and then HV (Table 2). Mean T1 values also differed between participant groups (WK: $F=7.9, p=0.001$, Cx: $F=6.9, p=0.003$, rep-Cx: $F=7.1, p=0.003)$. However, on paired comparisons, there was no difference in $\mathrm{T} 1$ results between HV and HF cohorts, while mean cortical T1 values were $122.4 \mathrm{~ms}(p=0.009)$ and $84.7 \mathrm{~ms}(p=0.02)$ greater in the Tx group compared to HV and HF groups, respectively. Medullary T1 values were also higher in Tx than HV (mean difference $129.1 \mathrm{~ms}, p=0.03$ ). There were no differences between groups on any cortical ROI for pCASL or ADC. Medullary pCASL values were significantly lower in Tx group compared to $\mathrm{HV}$ (mean difference $-35.7 \mathrm{ml} /$ $\min / 100 \mathrm{~g}, p=0.03$ ) and $\mathrm{HF}$ (mean difference $-48.4 \mathrm{ml} /$ $\min / 100 \mathrm{~g}, p=0.03)$.
Table 1 Patient demographics and clinical characteristics

\begin{tabular}{lllll}
\hline & All $(n=40)$ & HV $(n=10)$ & HF $(n=10)$ & Tx $(n=20)$ \\
\hline Age (years), median (IQR) & $56(39-63)$ & $43(30-58)$ & $62(54-70)$ & $51(38-61)$ \\
Male $(n, \%)$ & $28(70 \%)$ & $4(40 \%)$ & $7(70 \%)$ & $17(85 \%)$ \\
$\begin{array}{l}\text { eGFR (ml/min/1.73 m²), } \\
\text { median (IQR) }\end{array}$ & $60.0(37.7-76.7)$ & NA & $77.1(65.8-86.9)$ & $48.4(36.1-64.3)$ \\
\hline
\end{tabular}

$H V$ healthy volunteers, $H F$ heart failure, $T x$ renal transplant, $e G F R$ estimated glomerular filtration rate, $I Q R$ interquartile range 
Table 2 Comparison of results depending on region of interest, MRI sequence and participant group

\begin{tabular}{|c|c|c|c|c|c|c|c|c|}
\hline & \multicolumn{2}{|c|}{ All $(n=40)$} & \multicolumn{2}{|c|}{$\mathrm{HV}(n=20)$} & \multicolumn{2}{|c|}{$\mathrm{HF}(n=10)$} & \multicolumn{2}{|c|}{$\operatorname{Tx}(n=10)$} \\
\hline & Mean & SD & Mean & SD & Mean & SD & Mean & SD \\
\hline \multicolumn{9}{|c|}{ Volume $(\mathrm{ml})(n=39)$} \\
\hline Alternate slice & 195.8 & 56.8 & 147.8 & 30.9 & 170.6 & 45.3 & 230.1 & 49.2 \\
\hline Every slice & 197.5 & 57.4 & 149.0 & 31.3 & 170.8 & 44.9 & 232.6 & 49.3 \\
\hline \multicolumn{9}{|c|}{ T1 $(\mathrm{ms})(n=39)$} \\
\hline Whole kidney & 1772.8 & 131.4 & 1702.4 & 76.7 & 1696.5 & 85.7 & 1842.6 & 134.4 \\
\hline Cortex & 1630.2 & 102.0 & 1557.7 & 104.1 & 1595.5 & 80.1 & 1680.1 & 86.3 \\
\hline rep-Cx & 1606.1 & 114.4 & 1545.4 & 113.2 & 1543.8 & 71.1 & 1664.6 & 105.0 \\
\hline sup-Cx & 1655.6 & 119.5 & 1606.8 & 149.8 & 1600.0 & 93.4 & 1705.3 & 98.1 \\
\hline inf-Cx & 1639.0 & 103.7 & 1587.0 & 94.3 & 1590.0 & 113.3 & 1687.0 & 82.0 \\
\hline Med & 1975.8 & 74.9 & 1899.0 & 80.5 & 1940.2 & 71.3 & 2028.1 & 74.2 \\
\hline Cortex: Med & 0.83 & & 0.82 & & 0.82 & & 0.83 & \\
\hline \multicolumn{9}{|c|}{ pCASL $(\mathrm{ml} / \mathrm{min} / \mathbf{1 0 0} \mathrm{g})(n=37)$} \\
\hline Whole kidney & 181.7 & 56.6 & 187.5 & 58.4 & 161.6 & 47.1 & 190.2 & 60.7 \\
\hline Cortex & 221.0 & 80.0 & 235.1 & 79.3 & 175.7 & 59.5 & 239.3 & 84.3 \\
\hline rep-Cx & 260.8 & 91.4 & 271.8 & 93.5 & 228.4 & 92.3 & 273.3 & 90.4 \\
\hline sup-Cx & 196.4 & 75.9 & 230.5 & 77.9 & 160.4 & 54.2 & 197.4 & 79.6 \\
\hline inf-Cx & 225.2 & 105.2 & 213.8 & 91.1 & 161.8 & 84.4 & 269.2 & 107.5 \\
\hline Med & 95.8 & 41.8 & 108.8 & 45.1 & 121.5 & 31.5 & 73.0 & 45.9 \\
\hline Cortex: Med & 2.3 & & 2.2 & & 1.4 & & 3.3 & \\
\hline \multicolumn{9}{|c|}{$\operatorname{ADC}\left(\times 10^{-6} \mathrm{~mm}^{2} / \mathrm{s}\right)(n=28)$} \\
\hline Whole kidney & 1687.6 & 115.6 & 1687.2 & 97.4 & - & - & 1687.7 & 125.8 \\
\hline Cortex & 1678.1 & 111.4 & 1704.0 & 96.8 & - & - & 1665.8 & 118.1 \\
\hline rep-Cx & 1696.9 & 117.7 & 1719.9 & 158.6 & - & - & 1686.0 & 96.1 \\
\hline sup-Cx & 1686.3 & 144.2 & 1720.4 & 120.6 & - & - & 1670.1 & 154.5 \\
\hline inf-Cx & 1696.4 & 115.2 & 1700.5 & 111.8 & - & - & 1694.4 & 119.7 \\
\hline Med & 1671.9 & 82.5 & 1726.3 & 93.9 & - & - & 1646.1 & 77.2 \\
\hline Cortex: Med & 1.0 & & 1.0 & & & & 1.0 & \\
\hline
\end{tabular}

The standard deviation presented represents the spread of mean values. Volume measured by contouring alternate slices was similar to contouring every slice in all groups. Within each group, the values for whole kidney, cortical and medullary regions of interest were different for T1 and pCASL. In contrast, ADC values were similar for whole kidney, cortical and medullary regions of interest

$H V$ healthy volunteers, $H F$ heart failure, $T x$ renal transplant, $S D$ standard deviation, rep- $C x$ area of representative cortex, sup-Cx area of representative cortex at superior pole, inf- $C x$ area of cortex at inferior pole, Med medulla, $p C A S L$ pseudo-continuous arterial spin labelling, $A D C$ apparent diffusion coefficient

\section{Correlation between renal MRI and kidney function}

eGFR data were available for the 30 participants with heart failure or a renal transplant. There was no correlation between eGFR and renal volume, T1 or pCASL. There was a positive correlation between eGFR and ADC (Tx group only), with coefficients of: WK $0.47(p=0.04), \mathrm{Cx} 0.61$ $(p=0.006)$, rep-Cx $0.72(p=0.001)$, sup-Cx $0.45(p=0.05)$, inf-Cx $0.67(p=0.002)$ and Med $0.48(p=0.04)$.

\section{Discussion}

This study provides evidence to support the reproducibility of certain analysis techniques for renal MRI using commercially available analysis software. This is an essential step to allow studies exploring the clinical significance of functional renal MRI to report in confidence. Our data show that measurement of renal volume by contouring a localiser image is highly reproducible between observers. Contouring alternate slices, as opposed to every slice, results in a small reduction 
Fig. 2 Representative image (T1) displaying the regions of interest drawn for whole kidney $(\mathrm{WK})$, cortex $(\mathrm{Cx})$, representative cortex (rep-Cx), superior cortex (sup-Cx), inferior cortex (inf-Cx) and medulla (Med)

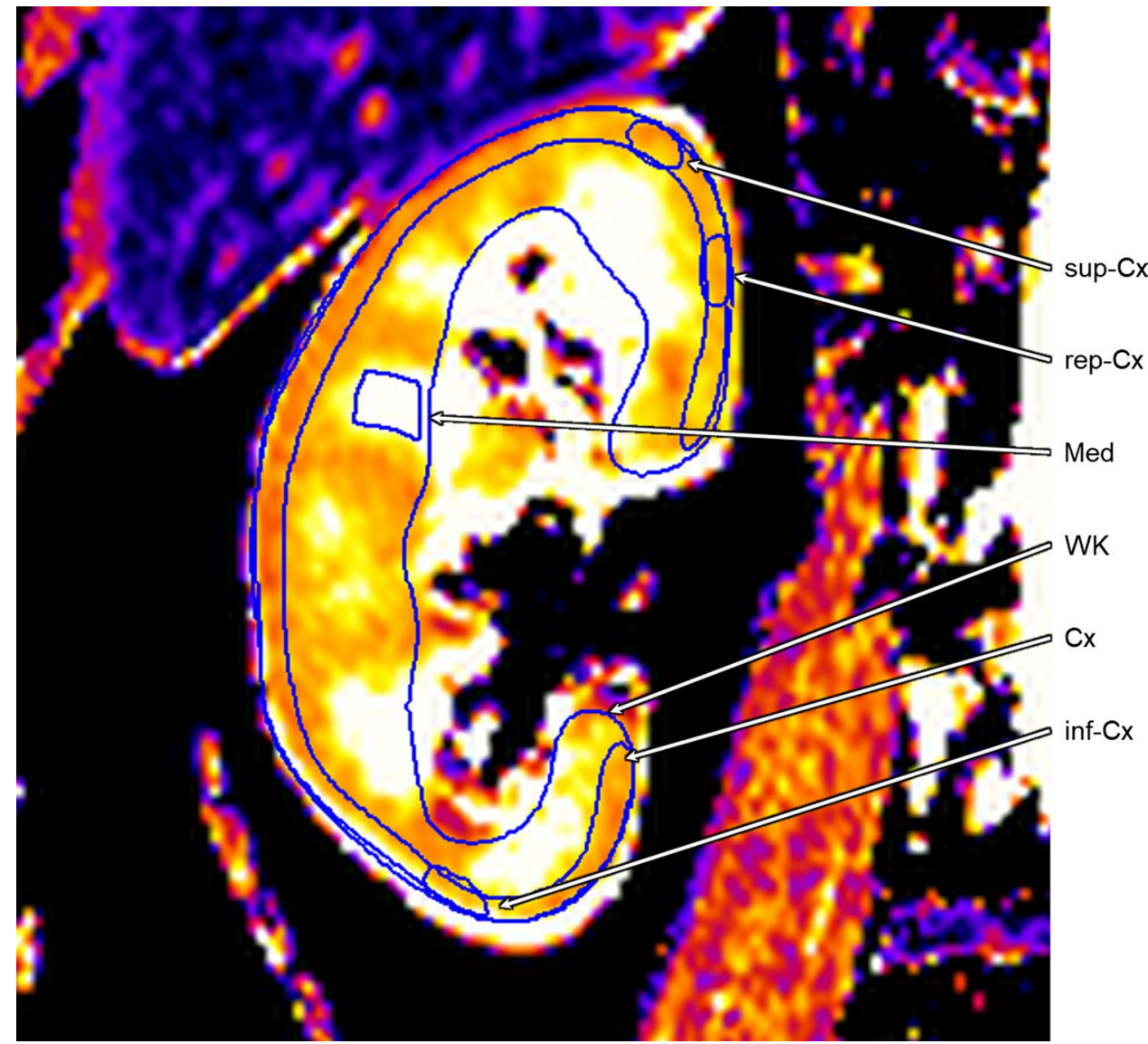

in measured volume with the advantage of improved efficiency. We believe the $1.6 \mathrm{ml}(0.8 \%)$ mean difference, in volume by contouring alternate slices, is clinically insignificant, but nevertheless we would advise consistency with whichever approach is chosen. Whilst automated contouring and volume calculation is being utilised by some centres
[16] and is likely to improve time efficiency, this approach is still to be externally validated and widely available. For T1, pCASL and ADC, WK ROIs are highly reproducible and commonly reported, but the mean value is derived from an unduly wide range of values, as evidenced by the fact on average the ROI SD represented between 17 and 55\% of the mean value in our cohort. We would argue this summary
Fig. 3 Bland-Altman plot comparing kidney volume as measured by contouring alternate slice versus every slice

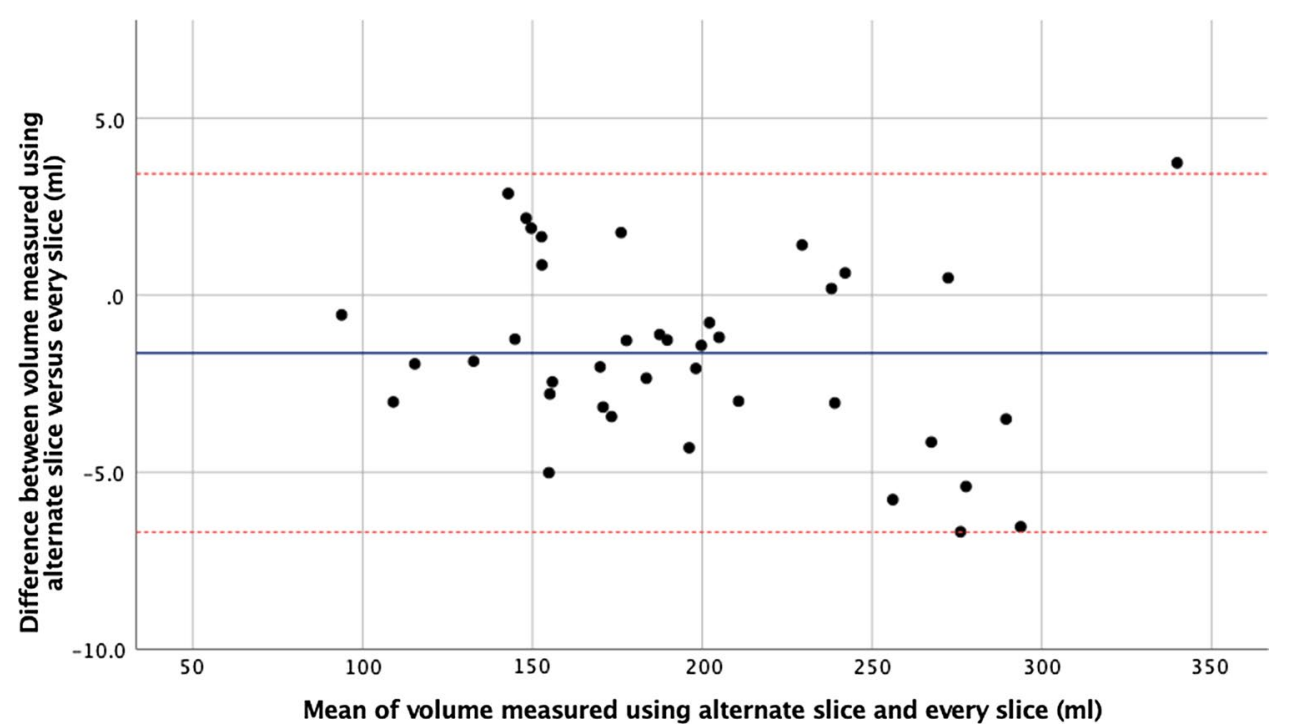


Table 3 Interobserver reproducibility by MRI sequence and analysis approach

\begin{tabular}{|c|c|c|}
\hline & $\mathrm{CoV}(\%)$ & ICC \\
\hline \multicolumn{3}{|c|}{ Volume $(\mathrm{ml})(n=39)$} \\
\hline Alternate slice & 6.5 & 0.97 \\
\hline Every slice & 6.7 & 0.96 \\
\hline \multicolumn{3}{|c|}{$\mathrm{T} 1(\mathrm{~ms})(n=39)$} \\
\hline Whole kidney & 1.0 & 0.97 \\
\hline Cortex & 1.2 & 0.97 \\
\hline rep-Cx & 2.0 & 0.95 \\
\hline sup-Cx & 3.2 & 0.96 \\
\hline inf-Cx & 2.5 & 0.86 \\
\hline Med & 2.6 & 0.87 \\
\hline \multicolumn{3}{|c|}{ pCASL $(\mathrm{ml} / \mathrm{min} / 100 \mathrm{~g})(n=37)$} \\
\hline Whole kidney & 7.0 & 0.90 \\
\hline Cortex & 10.3 & 0.93 \\
\hline rep-Cx & 14.2 & 0.86 \\
\hline sup-Cx & 19.1 & 0.69 \\
\hline inf-Cx & 14.6 & 0.92 \\
\hline Med & 29.6 & 0.73 \\
\hline \multicolumn{3}{|c|}{$\operatorname{ADC}\left(\times 10^{-6} \mathrm{~mm}^{2} / \mathrm{s}\right)(n=28)$} \\
\hline Whole kidney & 2.0 & 0.90 \\
\hline Cortex & 2.6 & 0.85 \\
\hline rep-Cx & 3.7 & 0.76 \\
\hline sup-Cx & 5.0 & 0.64 \\
\hline inf-Cx & 3.8 & 0.62 \\
\hline Med & 5.5 & 0.50 \\
\hline
\end{tabular}

Whole kidney and cortical ROIs were highly reproducible in all sequences

$\mathrm{CoV}$ coefficient of variation, ICC intraclass correlation coefficient, rep-Cx area of representative cortex, sup-Cx area of representative cortex at superior pole, inf-Cx area of cortex at inferior pole, Med medulla, $p C A S L$ pseudo-continuous arterial spin labelling, $A D C$ apparent diffusion coefficient

statistic is a crude representation of the physiological tissue, which we hope to describe and that cortical values may have more biological relevance, without unacceptable reduction in reproducibility. Indeed, for ADC, the correlation with renal function of cortical ROIs was stronger than for WK. When drawing a small ROI of representative cortex, prespecifying its location to be at either the superior (sup-Cx) or inferior (inf-Cx) pole did not improve reproducibility compared to a user-defined location and reduced the correlation with total cortex $(\mathrm{Cx})$ for T1 and pCASL. Furthermore, sup$\mathrm{Cx}$ and inf-Cx are theoretically more susceptible to artefact from respiratory movement in native kidneys compared to regions of lateral/medial cortex that would move in plane. We therefore advise that either $\mathrm{Cx}$ or rep-Cx be used preferentially, whenever cortical values are reported. Drawing an $\mathrm{ROI}$ for rep-Cx is likely to reduce analysis time compared to whole cortex and in this small sample, the correlation
Table 4 Table representing the spread of data from which the mean is calculated depending on region of interest and MRI sequence

\begin{tabular}{|c|c|c|}
\hline & ROI SD (mean) & $\begin{array}{l}\text { ROI SD as propor- } \\
\text { tion of mean value } \\
(\%)\end{array}$ \\
\hline \multicolumn{3}{|c|}{ T1 $(\mathrm{ms})(n=39)$} \\
\hline Whole kidney & 354.1 & 20.0 \\
\hline Cortex & 125.7 & 7.7 \\
\hline rep-Cx & 49.6 & 3.1 \\
\hline sup-Cx & 71.4 & 4.3 \\
\hline inf-Cx & 69.8 & 4.3 \\
\hline Med & 74.9 & 3.8 \\
\hline \multicolumn{3}{|c|}{ pCASL $(\mathrm{ml} / 100 \mathrm{~g} / \mathrm{min})(n=37)$} \\
\hline Whole kidney & 100.7 & 55.4 \\
\hline Cortex & 85.1 & 38.5 \\
\hline rep-Cx & 41.2 & 15.8 \\
\hline sup-Cx & 48.0 & 24.5 \\
\hline inf-Cx & 53.2 & 23.6 \\
\hline Med & 41.8 & 43.6 \\
\hline \multicolumn{3}{|c|}{$\operatorname{ADC}\left(\times 10^{-6} \mathrm{~mm}^{2} / \mathrm{s}\right)(n=28)$} \\
\hline Whole kidney & 289.5 & 17.2 \\
\hline Cortex & 169.6 & 10.1 \\
\hline rep-Cx & 71.5 & 4.2 \\
\hline sup-Cx & 105.8 & 6.3 \\
\hline inf-Cx & 85.9 & 5.1 \\
\hline Med & 84.7 & 5.1 \\
\hline
\end{tabular}

The ROI standard deviation is generated by the analysis software to represent the spread of values within each ROI. This table reports the mean ROI standard deviation for each sequence and displays it as a proportion of the mean value. The spread of data is larger for whole-kidney values, which includes cortical and medullary values as well as potential confounding data from vessels and renal pelvis. Conversely, the spread of data from the smaller ROIs of representative cortex may be uncharacteristically low if too small a ROI is drawn to be truly representative

$R O I$ region of interest, $S D$ standard deviation, rep-Cx area of representative cortex, sup-Cx area of representative cortex at superior pole, inf- $C x$ area of cortex at inferior pole, Med medulla, $p C A S L$ pseudocontinuous arterial spin labelling, $A D C$ apparent diffusion coefficient

between eGFR and ADC was greatest when rep-Cx was used. However, this is balanced against the lower ICC for rep-CX than Cx. Further studies are required to distinguish their benefits and we suggest that either $\mathrm{Cx}$ or rep-Cx can be used to report cortical values in the interim. Nevertheless, development of a harmonised approach across centres is vital to allow broader use of renal MRI in research and clinical settings [1].

While there was a significant correlation between ADC and eGFR, there was no association between renal volume, $\mathrm{T} 1$ and pCASL with renal function. Although this may generate scepticism with regards to the clinical relevance of these sequences, the development of MRI biomarkers is intended to provide physiologic and prognostic information 
additional to existing clinical measures, but further studies are needed to clarify this.

We performed a limited comparison of medullary values. Future studies may wish to analyse the medulla in more detail. Recent studies have reported measures of corticomedullary differentiation (CMD) using T1 and ADC and their correlation with clinical parameters [17-19]. These studies were well-conducted, but there is a risk of over interpreting the significance of cortico-medullary findings. Loss of CMD is a well-established, non-specific finding in CKD that is detectable on ultrasound, computed tomography and MRI [20]. Any observed association between eGFR and CMD on T1 or ADC may underplay the utility of MRI as a functional measurement and may instead detect a crude structural change that is prevalent in $\mathrm{CKD}$, and which can be measured in simpler ways.

The study is strengthened by its multi-parametric protocol across both healthy and diseased populations, including native and transplant kidneys yielding clinically meaningful results. The study has a number of limitations. Whilst we have shown these analyses to be reproducible, the clinical significance of any approach is not yet established. We did not assess R2* [also known as bloodoxygen-level-dependent (BOLD) imaging]. This parameter is recommended to be included in multi-parametric renal MRI protocols and its inclusion in this study would have been advantageous [1]. Only two observers reported each ROI for comparison of interobserver reproducibility. Kidney volume measurements were not compared with established 3D contrast-enhanced techniques, and further studies are required to assess the clinical relevance of kidney volume as measured by this approach. The current pCASL sequences utilise a fixed $\mathrm{T} 1$ value. We accept there may be advantages of using a measured $\mathrm{T} 1$ and we are exploring this for future studies. Other centres have developed efficient and accurate analysis methods, often using in-house developed software, which we are unable to replicate. For instance, a technique that uses a histogram to numerically segregate cortical from medullary values has been reported [4]. These analysis strategies require bespoke software which generally relies upon precise harmonisation of acquisition parameters to allow use out-with the centre in which they are developed. Nevertheless, comparison of results generated using this technique with the approaches detailed here would be interesting. The use of commercially available software in this study is strength. However, the license carries a cost and the software used is designed for cardiovascular analysis, such that we have applied many of the modules out-with their intended use. There is an urgent need for widely available software that is specifically designed for multi-parametric renal MRI analysis to advance the research and clinical application of renal MRI.

\section{Conclusion}

There are numerous strategies to analyse multi-parametric renal MRI with many centres using in-house bespoke software. The optimal approach is not yet known. These results provide justification for one approach using commercially available software. We suggest that kidney volume can be calculated by contouring alternate slices, rather than every slice, of a localiser scan albeit validation with $3 \mathrm{D}$ volume techniques is still required. For T1, pCASL and ADC, we suggest that whole kidney values, while highly reproducible, are used with caution given that the results represent a central value from an extremely wide range. Instead, manually delineated cortex or a small ROI of user-defined representative cortex can be used interchangeably in both native and transplant kidneys, with acceptable interobserver reproducibility. Clinical correlation of the results generated from this approach is eagerly awaited.

Acknowledgements Yuehui Tao (Siemens Healthcare, Erlangen, Germany) and Peter Weale (Siemens Healthcare, Erlangen, Germany) for assisting with application of the pCASL prototype sequence.

Authors' contribution All authors have contributed to this study. AJR, KAG, SAS and MMYL conceived the idea, designed the study and analysed the MRIs in conjunction with LZ. AJR, SAS and KAG wrote the manuscript, while LZ and MMYL prepared the figures. BK wrote the work-in-progress for pCASL. PBM, NS, GR, AR and RW, critically reviewed the manuscript.

Funding A.J.R. is supported by a Clinical Academic Training Fellowship from The Chief Scientist Office (Scotland) (CAF/18/02). K.A.G., P.B.M., A.R. and G.R. are supported by a grant from Kidney Research UK (KS_IN_002_20180913) and P.B.M., N.S., A.R. and M.M.Y.L. acknowledge research support from Boehringer Ingelheim.

\section{Compliance with ethical standards}

Conflict of interest The authors have a research agreement with Siemens for use of the renal pCASL work-in-progress sequence. Bernd Kuehn is an employee of Siemens Healthcare.

Ethical approval All procedures performed in studies involving human participants were in accordance with the ethical standards of the national research committee and with the 1964 Helsinki declaration and its later amendments.

Informed consent Informed consent was obtained from all individual participants included in the study.

Open Access This article is distributed under the terms of the Creative Commons Attribution 4.0 International License (http://creativeco mmons.org/licenses/by/4.0/), which permits unrestricted use, distribution, and reproduction in any medium, provided you give appropriate credit to the original author(s) and the source, provide a link to the Creative Commons license, and indicate if changes were made. 


\section{References}

1. Caroli A, Pruijm M, Burnier M, Selby NM (2018) Functional magnetic resonance imaging of the kidneys: where do we stand? The perspective of the European COST Action PARENCHIMA. Nephrol Dial Transplant 33(suppl_2):ii1-ii3

2. Selby NM, Blankestijn PJ, Boor P, Combe C, Eckardt KU, Eikefjord E, Garcia-Fernandez N, Golay X, Gordon I, Grenier N, Hockings PD, Jensen JD, Joles JA, Kalra PA, Kramer BK, Mark PB, Mendichovszky IA, Nikolic O, Odudu A, Ong ACM, Ortiz A, Pruijm M, Remuzzi G, Rorvik J, de Seigneux S, Simms RJ, Slatinska J, Summers P, Taal MW, Thoeny HC, Vallee JP, Wolf M, Caroli A, Sourbron S (2018) Magnetic resonance imaging biomarkers for chronic kidney disease: a position paper from the European Cooperation in Science and Technology Action PARENCHIMA. Nephrol Dial Transplant 33(suppl_2):ii4-ii14

3. Martirosian P, Klose U, Mader I, Schick F (2004) FAIR trueFISP perfusion imaging of the kidneys. Magn Reson Med 51(2):353-361

4. Cox EF, Buchanan CE, Bradley CR, Prestwich B, Mahmoud H, Taal M, Selby NM, Francis ST (2017) Multiparametric renal magnetic resonance imaging: validation, interventions, and alterations in chronic kidney disease. Frontiers Physiol 8:696

5. Gillis K, McComb C, Foster J, Taylor A, Patel R, Morris S, Jardine A, Schneider M, Roditi G, Delles C, Mark P (2014) Interstudy reproducibility of arterial spin labelling magnetic resonance imaging for measurement of renal perfusion in healthy volunteers at 3 Tesla. BMC Nephrol 15(1):1-10

6. Gillis KA, McComb C, Patel RK, Stevens KK, Schneider MP, Radjenovic A, Morris ST, Roditi GH, Delles C, Mark PB (2016) Non-contrast renal magnetic resonance imaging to assess perfusion and corticomedullary differentiation in health and chronic kidney disease. Nephron 133(3):183-192

7. Levey AS, Stevens LA, Schmid CH, Zhang YL, Castro AF III, Feldman HI, Kusek JW, Eggers P, Van Lente F, Greene T, Coresh J (2009) A new equation to estimate glomerular filtration rate. Ann Intern Med 150(9):604-612

8. Look DC, Locker DR (1970) Time saving in measurement of NMR and EPR relaxation times. Rev Sci Instrum 41(2):250-251

9. Messroghli DR, Radjenovic A, Kozerke S, Higgins DM, Sivananthan MU, Ridgway JP (2004) Modified look-locker inversion recovery (MOLLI) for high-resolution T1 mapping of the heart. Magn Reson Med 52(1):141-146

10. Wolf M, de Boer A, Sharma K, Boor P, Leiner T, Sunder-Plassmann G, Moser E, Caroli A, Jerome NP (2018) Magnetic resonance imaging T1- and T2-mapping to assess renal structure and function: a systematic review and statement paper. Nephrol Dial Transplant 33(suppl_2):ii41-ii50

11. Xue H, Greiser A, Zuehlsdorff S, Jolly MP, Guehring J, Arai AE, Kellman P (2013) Phase-sensitive inversion recovery for myocardial T1 mapping with motion correction and parametric fitting. Magn Reson Med 69(5):1408-1420

12. Silva AC, Kim SG (1999) Pseudo-continuous arterial spin labeling technique for measuring $\mathrm{CBF}$ dynamics with high temporal resolution. Magn Reson Med 42(3):425-429

13. Odudu A, Nery F, Harteveld AA, Evans RG, Pendse D, Buchanan CE, Francis ST, Fernández-Seara MA (2018) Arterial spin labelling MRI to measure renal perfusion: a systematic review and statement paper. Nephrol Dial Transplant 33(suppl_2):ii15-ii21

14. Caroli A, Schneider M, Friedli I, Ljimani A, De Seigneux S, Boor P, Gullapudi L, Kazmi I, Mendichovszky IA, Notohamiprodjo M, Selby NM, Thoeny HC, Grenier N, Vallée J-P (2018) Diffusion-weighted magnetic resonance imaging to assess diffuse renal pathology: a systematic review and statement paper. Nephrol Dial Transplant 33(suppl_2):ii29-ii40

15. Bland JM, Altman DG (1986) Statistical methods for assessing agreement between two methods of clinical measurement. Lancet (Lond, Engl) 1(8476):307-310

16. Kim Y, Ge Y, Tao C, Zhu J, Chapman AB, Torres VE, Yu ASL, Mrug M, Bennett WM, Flessner MF, Landsittel DP, Bae KT, Consortium for Radiologic Imaging Studies of Polycystic Kidney Disease (CRISP) (2016) Automated segmentation of kidneys from MR images in patients with autosomal dominant polycystic kidney disease. Clin J Am Soc Nephrol 11(4):576-584

17. Friedli I, Crowe LA, Berchtold L, Moll S, Hadaya K, de Perrot T, Vesin C, Martin PY, de Seigneux S, Vallée JP (2016) New magnetic resonance imaging index for renal fibrosis assessment: a comparison between diffusion-weighted imaging and T1 mapping with histological validation. Sci Rep 6:30088

18. Buchanan CE, Mahmoud H, Cox EF, McCulloch T, Prestwich BL, Taal MW, Selby NM, Francis ST (2019) Quantitative assessment of renal structural and functional changes in chronic kidney disease using multi-parametric magnetic resonance imaging. Nephrol Dial Transplant. https://doi.org/10.1093/ndt/gfz129

19. Pruijm M, Milani B, Pivin E, Podhajska A, Vogt B, Stuber M, Burnier M (2018) Reduced cortical oxygenation predicts a progressive decline of renal function in patients with chronic kidney disease. Kidney Int 93(4):932-940

20. Semelka RC, Corrigan K, Ascher SM, Brown JJ, Colindres RE (1994) Renal corticomedullary differentiation: observation in patients with differing serum creatinine levels. Radiology 190(1):149-152

Publisher's Note Springer Nature remains neutral with regard to jurisdictional claims in published maps and institutional affiliations. 